\title{
Intelligent Decision Model Based on Optimized Simulated Annealing Algorithm
}

\author{
Hui Xia \\ Software College,Shenyang Normal university, Shenyang, 110034, China \\ freund_xia@126.com
}

\begin{abstract}
Keywords: decision-theoretic; rough set model; simulated annealing algorithm;cost function Abstract. Rough set theory can be applied to rule induction.The rough set theory approximates a concept by three regions, namely, the positive,boundary and negative regions.Because of making decision by rough set constructing model, cost of the decision should be considered, we will propose an optimized simulated annealing algorithm(OSAA), which is an optimized algorithm for a minimum cost attribute reduction. A heuristic approach combined with the coarse-grained parallel algorithm are proposed to solve the problem of optimization DTRS.Experiments designed for the optimization simulated annealing algorithm and adaptive learning method are in comparing with the running time and decision-making cost. The optimization representation can bring some new insights into the research on decision-theoretic rough set model.
\end{abstract}

\section{Introduction}

In Pawlak' s rough set theory [1] and its probabilistic generalizations [9,10], all decision classes are treated as the same in the interpretation and applications of approximations and three regions. In other words, the same pair of thresholds are used to define the positive, negative and boundary regions. As a natural extension to these studies, rough set approximations for multi-class decision problems using different pairs of thresholds have been discussed in several literatures.

The three-way decision rules come closer to the philosophy of the rough set theory, namely, representing a concept usingthree regions instead of two [1]. This three-way decision scheme has not been considered explicitly in other theories of machine learning and rule induction, although it has been studied in other fields. By considering three-way decision rules, one may appreciate the true and unique contributions of rough set theory to machine learning and rule induction. With the introduction of the notion of three-way decisions, there is a new avenue of research for rough set theory. The cost-based analysis of three-way decisions brings the theory closer to real-world applications where costs play an indispensable role[2,3].

In this paper, we will overcome the inherent seriality of classic simulated annealing algorithm and further combine it with downhill method and synthesize a variety of optimization methods to obtain a large scalable parallel effect, so as to improve the convergence speed of the algorithm, Value and parameter selection, and finally get the optimal decision threshold, so as to improve the practical application efficiency of Rough Set Decision Model.

\section{DTRS Model}

According to Bayesian decision-making flow and DTRS model [11], a decision-making optimization system model is constructed, and the relationship between the decision-making threshold factor and decision cost is constructed.

First, a decision model is created and the decision thresholds are expressed according to the minimum cost of the decision model. Then, the decision rules are deduced according to the Bayesian decision process. Then, the cost model is represented by some reduction rules. Finally, according to the set conditions Decision expression and given threshold, the cost function of the three decision rules of positive rule, boundary rule and negative rule is obtained, and the three decision cost functions are accumulated to obtain the total cost function. 


\section{1) DTRS Decision model parameters}

$$
\begin{aligned}
& \alpha=\frac{\left(\lambda_{P N}-\lambda_{B N}\right)}{\left(\lambda_{P N}-\lambda_{B N}\right)+\left(\lambda_{B P}-\lambda_{P P}\right)} \\
& \beta=\frac{\left(\lambda_{B N}-\lambda_{N N}\right)}{\left(\lambda_{B N}-\lambda_{N N}\right)+\left(\lambda_{N P}-\lambda_{B P}\right)} \\
& \gamma=\frac{\left(\lambda_{P N}-\lambda_{N N}\right)}{\left(\lambda_{P N}-\lambda_{N N}\right)+\left(\lambda_{N P}-\lambda_{P P}\right)}
\end{aligned}
$$

Where $\lambda_{P P}, \lambda_{B P}$ and $\lambda_{N P}$ denote the price after taking P, B, $\mathrm{N}$ action respectively when the object belongs to a state $\mathrm{C}, \lambda_{P N}, \lambda_{B N}$ and $\lambda_{N N}$ denote the price after taking P, B, N action respectively when the object belongs to a state.

\section{2) A Cost Function Model for Decision}

According to DTRS theory,we can get:

$$
R\left(a_{i} \mid x\right)=\sum_{j=1}^{x} \lambda\left(a_{i} \mid \omega_{j}\right) \cdot p\left(\omega_{j} \mid x\right)
$$

The decision thresholds expression $\alpha, \beta, \gamma$ are obtained according to the Bayesian decision theory,Let $\lambda_{P P}=\lambda_{N N}=0$ according to the probability rough set model (the correct decision cost is zero), Finally, the decision-making cost of the decision-making threshold $\alpha, \beta, \gamma$ is obtained,which is $\operatorname{COST}(\alpha, \beta, \gamma)$, The cost of decision-making process shown in Figure 1:

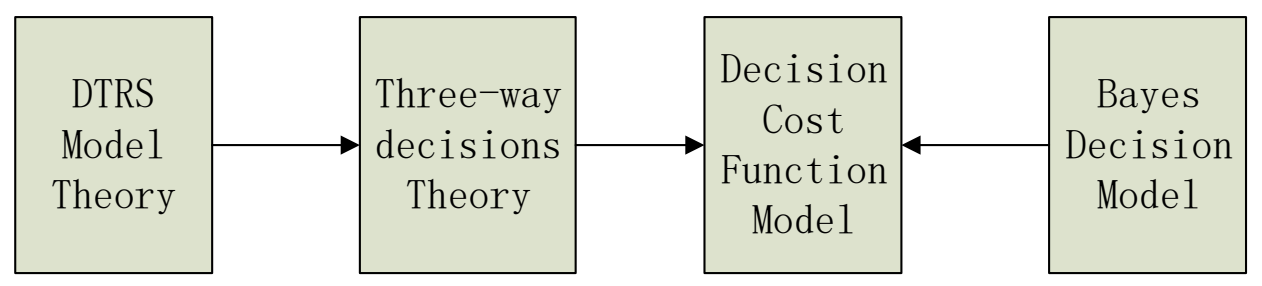

Fig 1.DTRS decision cost function model representation flow

\section{3) Pawlak reduction theory model[4-6]}

In Pawlak's rough set model [1], informations about a finite set of objects are represented in an information table with a finite set of attributes. Formally, an information table can be expressed as:

$$
S=\left(U, A t=C \bigcup\{D\},\left\{V_{a} \mid a \in A t\right\},\left\{I_{a} \mid a \in A t\right\}\right)
$$

The attribute $R \subseteq C$ is a reduction of the attribute of set $C$ with respect to $\mathrm{D}$, which satisfies two conditions (a necessary and sufficient condition):

sufficient condition: $\operatorname{POS}_{\pi_{R}}\left(\pi_{D}\right)=P O S_{\pi_{C}}\left(\pi_{D}\right)$

necessary condition: $P O S_{\pi_{R-\{a\}}}\left(\pi_{D}\right) \neq P O S_{\pi_{C}}\left(\pi_{D}\right)$ where $a \in R$

\section{An Optimized Simulated Annealing Algorithm for Coarse - grained Parallel Computation}

Optimizing the basic idea of simulated annealing algorithm: OSAA (Optimization Simulated Annealing Algorithm) decision-making model is based on the original classic simulated annealing model to do significant parallelization improvement, combined with the downhill method of rapid convergence characteristics, it is a scalable Large - scale parallel computing method.

1) Parallel computing model framework

The MPS + OpenMP multi-granularity computer cluster is chosen to optimize the computing 
granularity of the algorithm and improve the parallel speedup.

Construction of Simulated Annealing Model Initialization. To set the initialization temperature: $t_{0}=\frac{f_{\min }-f_{\max }}{\ln P_{0}}$, where $f_{\min }$ is the minimum value of the fitness function, $f_{\max }$ is the fitness function maximum.

2) Condition Analysis of Critical Point of Model

Model selection minimum point principle and condition analysis. The annealing condition is selected according to the simulated annealing principle. For any value, the simulated annealing algorithm may choose the worst point: $e^{\frac{-\left(f_{N(X)}-f_{X}\right)}{t_{i}}}>\sigma,(0<\sigma<1)$.

3) Build the OSAA model

According to the classical annealing algorithm, initialize the OSAA schedule, construct a fitness function $f(R), \mathrm{R}$ is the reduction attribute set, set an initial temperature $t_{0}$, record the number of annealing $\mathrm{N}$, and determine the current solution by comparing $\operatorname{COST}_{N E W}$ and $C O S T_{O L D}$. After each comparison, We must use a probability factor to call the down function $\operatorname{DownHill}\left(\operatorname{COST}_{c}\right)$, the design model strategy: multiple annealing without progress to find the optimal solution to take down the high probability, so as to truly find the global optimal solution, rather than the local optimal solution, And to communicate with other computing nodes to compare each other's optimal solution, so as to achieve the effect of parallel computing.

\section{The Evaluation System of Decision - Making Rough Set Model}

Trust standard: Confidence $\left([x] \rightarrow D_{i}\right)=\frac{\left|[x] \cap D_{i}\right|}{|[x]|}=P\left(D_{i} \mid[x]\right)$

Coverage standard: coverage $\left([x] \rightarrow D_{i}\right)=\frac{\left|[x] \cap D_{i}\right|}{\left|D_{i}\right|}=P\left([x] \mid D_{i}\right)$

Cost standard:

$$
\begin{aligned}
& R\left([x] \rightarrow{ }_{P} D_{i}\right)=\lambda_{P N}+\left(\lambda_{P P}-\lambda_{P N}\right) \text { Confidence }\left([x] \rightarrow{ }_{P} D_{i}\right) \\
& R\left([x] \rightarrow{ }_{B} D_{i}\right)=\lambda_{B N}+\left(\lambda_{B P}-\lambda_{B N}\right) \text { Confidence }\left([x] \rightarrow{ }_{B} D_{i}\right)
\end{aligned}
$$

\section{Test results}

Experiments were carried out using several sets of data from UCI (University of California-Irvine) [7] datasets. The corresponding algorithms were integrated and implemented on the WEKA platform [8]. The experiments were conducted to verify the following two aspects of decision theory:

1) Optimized simulated annealing algorithm (OSAA) and adaptive algorithm are compared, respectively, from the running time, minimum cost, algorithm complexity comparison;

2) the cost of optimization of the minimum goal of the simple attribute algorithm validation.

The experimental equipment used is IntelCore i7-3770 processor, 8G of memory, 64-bit Windows10 operating system, the system algorithm is based on the Weka platform. Platform experimental data is from the UCI database of five data sets.

The results of this experiment are shown in Table 1, and the decision-making cost of decision-making based on two algorithms, Alcofa and Simulated Annealing Algorithm, is used. 
Table 2 shows the average running cost of the adaptive algorithm and simulated annealing algorithm Time (in ms).

Table.1 Comparison of running time of two algorithms

\begin{tabular}{ccc}
\hline \multirow{2}{*}{ Data Set } & \multicolumn{2}{c}{ Run time $(\mathrm{ms})$} \\
\cline { 2 - 3 } & Alcofa & OSAA \\
\hline bands & 33.4 & 7.2 \\
Credit & 59.2 & 5.1 \\
Monk1 & 7.2 & 5.8 \\
Monk2 & 17.1 & 1.9 \\
Musk & 24.4 & 13.9 \\
\hline
\end{tabular}

Table.2 Comparison of decision cost between two algorithms

\begin{tabular}{ccc}
\hline \multirow{2}{*}{ Data Set } & \multicolumn{2}{c}{ Decision cost } \\
\cline { 2 - 3 } & Alcofa & OSAA \\
\hline bands & 42.44 & 1.98 \\
Credit & 3.23 & 1.04 \\
Monk1 & 45.22 & 1.49 \\
Monk2 & 7.22 & 1.89 \\
Musk & 2.98 & 0.96 \\
\hline
\end{tabular}

\section{Conclusion}

In this paper, an optimized DTRS model is proposed. The optimal criterion is based on the minimum decision cost. The optimized model can learn cost function and decision threshold without prior knowledge, and has higher efficiency compared with the previous learning method. DTRS model algorithm proposed in this paper has good validity and shorter running time than the adaptive algorithm, and the learning threshold can get a smaller decision-making risk.

\section{References}

[1] Z. Pawlak, Rough Sets, Theoretical Aspects of Reasoning about Data, Kluwer Academic Publishers, Dordrecht, 1991.

[2] Y. Li, C. Zhang, J.R. Swan, An information filtering model on the Web and its application in JobAgent, Knowledge-Based Systems 13 (2000) 285-296.

[3] J.T. Yao, J.P. Herbert, Web-based support systems based on rough set analysis, in: Proceedings of RSEISP'07, LNAI 4585, 2007, pp. 360-370

[4] Kai Zheng, Jie Hu, Zhenfei Zhan,et al.An enhancement for heuristic attribute reduction algorithm in rough set[J].Expert Systems with Applications, 2014,41(15):6748-6754.

[5] Changzhong Wang, Mingwen Shao, Baiqing Sunc,et al. An improved attribute reduction scheme with covering basedrough sets[J].Applied Soft Computing, 2015,26:235-243.

[6] Bing Li, Tommy W.S.Chow,Peng Tang. Analyzing rough set based attribute reductions by extension rule[J].Neurocomputing,2014,123:185-196.

[7] UCI Machine Learning Repository, <http://archive.ics.uci.edu/ml/>.

[8] http://www.cs.waikato.ac.nz/ ml/weka/

[9] J.P.Herbert,J.T.Yao,Game-theoreticriskanalysisindecision-theoreticroughsets, in:ProceedingsofR SKT'08,LectureNotesinComputerScience,vol.5009,2008, pp. 132-139.

[10]J.P. Herbert, J.T. Yao, Game-theoretic rough sets, Fundamenta Informaticae - Advances in Rough Set Theory 108 (3-4) (2011) 267-286.

[11]Nouman Azam, JingTao Yao.Analyzing uncertainties of probabilistic rough set regions with game-theoretic rough sets[J], International Journal of Approximate Reasoning,2014,55:142-155. 